Ann. Parasitol. Hum. Comp., 1991, $66: \mathrm{n}^{\circ} 3,105-108$.

Mémoire.
Mots-clés : Monogenea. Ancyrocephalidae. Enterogyrus. Endoparasite. Cichlidae. Endémisme. Lac de cratère. Cameroun. Spécificité parasitaire.

Key-words: Monogenea. Ancyrocephalidae. Enterogyrus. Endoparasite. Cichlidae. Endemism. Crater lake. Cameroon. Parasitic specificity.

\title{
ENTEROGYRUS BAROMBIENSIS N. SP. (MONOGENEA, ANCYROCEPHALIDAE) PARASITE STOMACAL DE TROIS CICHLIDAE ENDÉMIQUES DU LAC DU CRATÈRE BAROMBI MBO (CAMEROUN)
}

\author{
C. F. BILONG BILONG*, E. BIRGI**, L. EUZET**
}

RÉSUMÉ

Description d'une nouvelle espèce : Enterogyrus barombiensis (Monogenea, Ancyrocephalidae) parasite stomacal de trois Cichlidae endémiques du lac de cratère Barombi Mbo au Cameroun. Discussion sur la spécificité parasitaire de ce Monogène.

Summary: Enterogyrus barombiensis n. sp. (Monogenea Ancyrocephalidae) stomach parasite of three endemic cichlidae from the crater lake Barombi Mbo (Cameroon).

Enterogyrus barombiensis a new Monogenea Ancyrocephalidae, stomach parasite of three endemic Cichlidae from the crater lake
Barombi Mbo in Cameroon, is described. The problem of its specificity is discussed herein.

\section{INTRODUCTION}

Le lac Barombi Mbo est un lac de cratère situé à $50 \mathrm{~km}$ au N-NE du Mont Cameroun, à $9^{\circ} 22^{\prime} \mathrm{N}$ et $4^{\circ} 38^{\prime} \mathrm{N}$ dans la province du Sud-Ouest. Il s'étend au sommet d'une petite montagne de 290 à $308 \mathrm{~m}$ d'altitude et couvre une superficie de 453 hectares avec $110 \mathrm{~m}$ de profondeur maximale, $2,5 \mathrm{~km}$ de diamètre. Sur le versant Sud-Est, il existe un déversoir par où s'écoule (pendant la saison des pluies) l'eau du lac, vers la Kumba River, affluent du fleuve Mungo. Le lac est habité par une importante faune ichtyologique qui a permis le développement d'une pêche artisanale active. Parmi ces poissons, Trewavas et al., 1972, signalent onze espèces de Cichlidae endémiques. Dans le cadre de notre analyse des Monogènes parasites des poissons des eaux douces du Cameroun, nous avons étudié les Cichlidae endémiques de ce plan d'eau. En effet, le parasitisme de telles populations naturelles isolées n'a été que rarement abordé.

* Laboratoire de Biologie Générale, Faculté des Sciences, BP 812, Yaoundé, Cameroun.

** Laboratoire de Parasitologie Comparée, USTL, Place E.-Bataillon, 34095 Montpellier, Cedex 5, France.

Accepté le : 25 mai 1991.

\section{MATÉRIEL ET MÉTHODES}

Les poissons sont capturés à la nasse par les pêcheurs locaux. Ils sont conservés dans des récipients remplis d'eau du lac et transportés au laboratoire dans des bacs isothermes munis d'aérateurs.

Après ouverture médio-ventrale du poisson, le système digestif est séparé par incision antérieure au niveau de l'œsophage et postérieure au niveau de l'anus.

L'estomac isolé est ouvert longitudinalement et observé sous la loupe binoculaire. Les Monogènes, solidement accrochés par leur hapteur à la paroi stomacale, sont détachés. Certains individus vivants sont déposés dans une goutte d'eau et observés entre lame et lamelle. D'autres sont fixés au mélange de Malmberg (1970) (picrate d'ammonium-glycérine). Les préparations sont lutées à l'aide du lut de Rondeau du Noyer. Les individus sont dessinés à la chambre claire. Les mensurations des crochets sont celles définies par Gussev (in Bykowskaya-Pavloskaya, 1962) (fig. 1). La dénomination et la numérotation des crochetons sont celles suggérées par Llewellyn (1963) et adoptée à ICOPA IV, Varsovie, 1978 (Euzet et Prost, 1981).

Les mesures sont données en micromètre $(\mu \mathrm{m})$ de la manière suivante : moyenne (minimum-maximum).

Enterogyrus barombiensis n. sp. (fig. 2 et 3).

Hôtes : Pungu maclareni Trewavas, Green and Corbet, 1972; Stomatepia pindu Trewavas, Green and Corbet, 1972; Konia eisentrauti Trewavas, Green and Corbet, 1972.

Habitat : estomac.

Localité : lac Barombi Mbo à Kumba, Cameroun. 

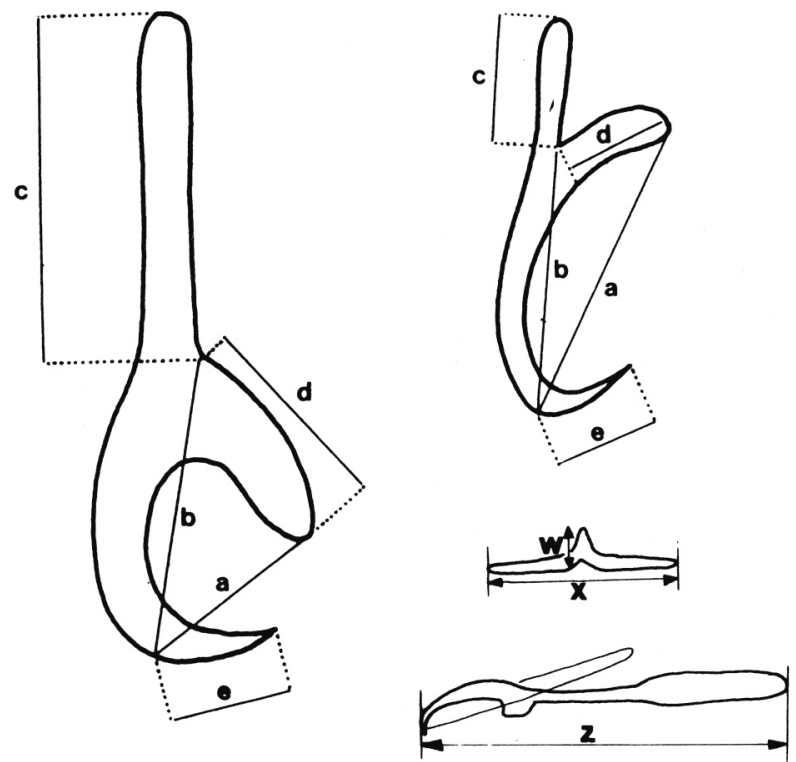

Fig. 1. - Nomenclature pour la mensuration des pièces haptoriales des Monogènes Monopisthocotyles ( $d$ 'après BykhowskayaPavlovskaya et coll., 1962), adaptée aux Enterogyrus par Bilong Bilong et al., 1989. a : longueur totale des crochets; b : longueur de la lame; c : longueur du manche; $d$ : longueur de la garde; e : longueur de la pointe; w : largeur de la pièce (barre) transversale; $\mathrm{x}$ : longueur de la pièce (barre) transversale; $\mathrm{z}$ : longueur du crocheton.

Matériel étudié : 6 individus sur le vivant ; 35 colorés au liquide de Malmberg.

Types : déposés au MNHN Paris, sous les numéros 111HF Tk7 (holotype) et 112HF Tk8 (paratype).

Les individus adultes mesurent 525,7 (450-600) de longueur et $115,7(100-130)$ de largeur au niveau du testicule. Une constriction postérieure marque la limite entre le hapteur et le reste du corps. Ce hapteur porte 14 crochetons, 2 paires de hamuli et une barre transversale ventrale. Sa morphologie est identique à celle décrite chez Enterogyrus coronatus et E. foratus Pariselle et al. Il comprend deux parties :

- un « pédoncule » postérieur contenant :

- les hamuli ( 2 dorsaux et 2 ventraux),

- une barre transversale ventrale,

- les crochetons ventraux I et II;

— un « bulbe » antérieur dont la largeur supérieure à celle du corps, est le double de celle du pédoncule et au niveau duquel on observe - disposés en un cercle équatorial - les crochetons dorsaux III et IV et ventraux V à VII. La pointe de tous ces crochetons est dirigée antérieurement.

Le hapteur mesure 132,9 (110-170) de longueur et 108,3 (90-130) de largeur au niveau du bulbe.

Les hamuli dorsaux (fig. 2) sont robustes et du type E. melenensis Bilong Bilong et al., 1989 avec un manche long, rectiligne, une garde courte dirigée vers la lame qui est régulièrement courbée. Chaque hamuli porte un filament très sclérifié coiffant la lame. Ces crochets mesurent: $a=10,2$ (9-12); $b=16,7$ (16-18); $\mathrm{c}=12,2(11-13) ; \mathrm{d}=8,1(7-9) ; \mathrm{e}=4,5(4-5) ; \mathrm{b}+\mathrm{c}=29,4$ (27-31).

Les hamuli ventraux, plus petits, ont un manche plus étroit que la garde. Ils mesurent: $a=11,8$ (11-13); $b=10,9$ (10-12); $\mathrm{c}=6,0(5-7) ; \mathrm{d}=5,0(4-6) ; \mathrm{e}=4,0(3-5) ; \mathrm{b}+\mathrm{c}=16,9(16-17)$.

La barre transversale ventrale, très mince (1 d'épaisseur) a la forme d'un V. Elle mesure 16,9 (16-19) de longueur totale. Les 14 crochetons, tous semblables, ont 11,9 (11-13) de longueur.

L'appareil copulateur mâle est caractérisé par un pénis situé sur le plan médian, dans le quart antérieur du corps. C'est un tube élargi (4-5) à la base, qui dessine 6 spires courtes serrées dans sa partie proximale, puis 2 spires lâches dans sa région médiane et 4 très serrées dans sa portion distale; soit une spiralisation qui peut être schématisée par la formule « 6-2-4» (fig. 3). Ce pénis mesure 46,7 (42-52) de longueur. Le vagin non sclérifié n'a pas été observé. L'anatomie de l'animal correspond à celle du genre Enterogyrus Paperna, 1963, telle que nous l'avons revue (Bilong Bilong et al., 1989).

\section{DISCUSSION}

A ce jour une dizaine d'espèces sont décrites dans le genre Enterogyrus en Afrique et en Asie. Elles sont toutes inféodées à des poissons de la famille des Cichlidae. Elles se distinguent par la taille de leurs pièces haptoriales et la morphologie de leur appareil copulateur. Guégan (1990) estime que les affinités phylétiques des Monogènes Dactylogyridea devraient être recherchées plus dans l'anatomie du système génital et la morphologie des pièces copulatrices que dans la structure des organes d'attachement. Les individus récoltés chez les Cichlidae endémiques du lac Barombi Mbo ont un pénis dont la spiralisation «6-2-4 » ne se retrouve chez aucune autre espèce d'Enterogyrus. En outre la taille des pièces haptoriales est généralement plus petite que chez les espèces connues. Pour ces raisons, nous estimons que ce parasite appartient à une espèce nouvelle que nous proposons de nommer Enterogyrus barombiensis n. sp.

Les Enterogyrus décrits à ce jour ont en général une spécificité de type oöoxène sauf $E$. cichlidarum Paperna, 1963 parasite de Tilapia zillii et signalé chez $T$. aurea et T. nilotica Paperna, 1979.

Pour la première fois, nous rencontrons un Enterogyrus parasite de trois espèces de Cichlidae appartenant à trois genres différents. Il s'agit ici d'une véritable euryxénie. Il faut cependant noter que cela s'observe dans un lac de cratère (biocénose relativement restreinte) où s'est manifestée une très forte endémicité de poissons hôtes. Cet isolement est peut-être à l'origine de cette euryxénie. En effet, nous avons récolté chez ces trois poissons un seul Cichlidogyrus du type $C$. tilapiae Paperna, 1960 sur les branchies; un Urogyrus n. g. (Bilong Bilong, à paraitre) dans la vessie urinaire et Enterogyrus barombiensis dans l'estomac.

Le parasite de la vessie (Urogyrus n. g.) a été de plus observé chez Nanochromis caudifasciatus, Chromidotilapia batesi, Tilapia spp. dans d'autres régions au Cameroun.

Par contre, E. barombiensis n'a pas été retrouvé chez d'autres Cichlidae dans les bassins hydrographiques camerounais prospectés. 

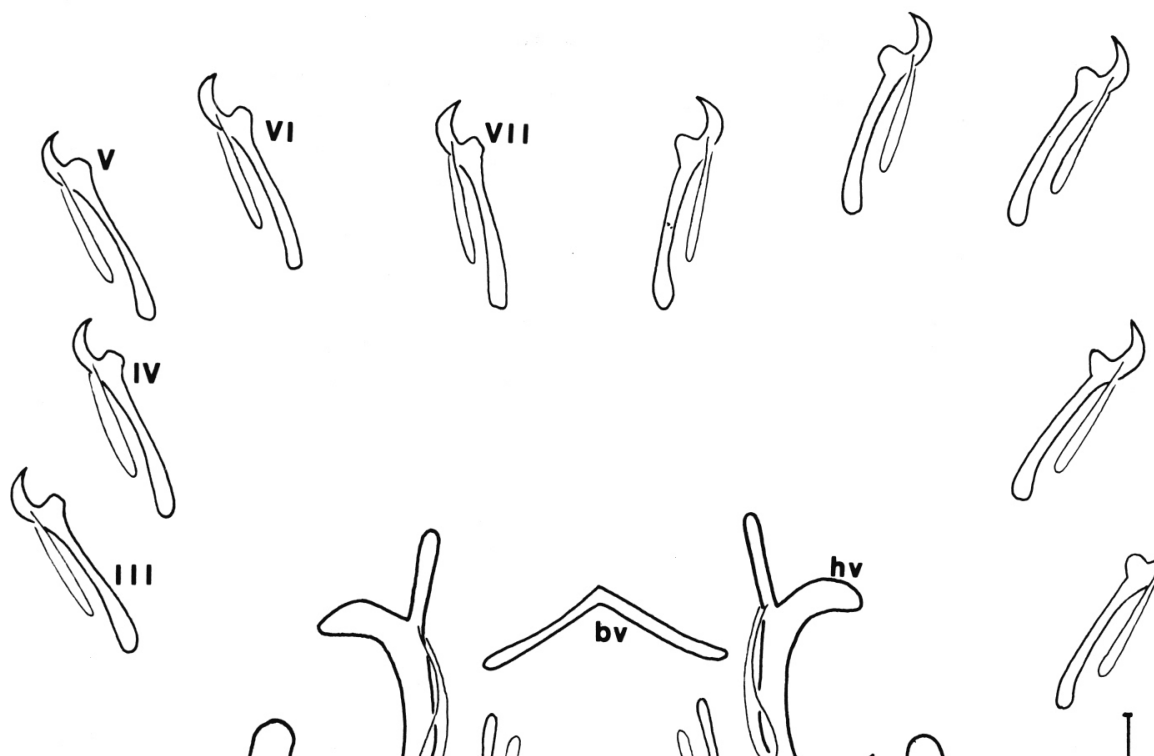

\begin{abstract}
hamuli dorsal; hv : hamuli ventral; I et II : crochetons ventraux (dans le pédoncule); III et IV : crochetons dorsaux (dans le bulbe); V à VII : crochetons ventraux (dans le bulbe).
\end{abstract}

FIG. 2. - Pièces sclérifiées du hapteur. bv : barre transversale ventrale; hd :

Fig. 3. - Pénis.

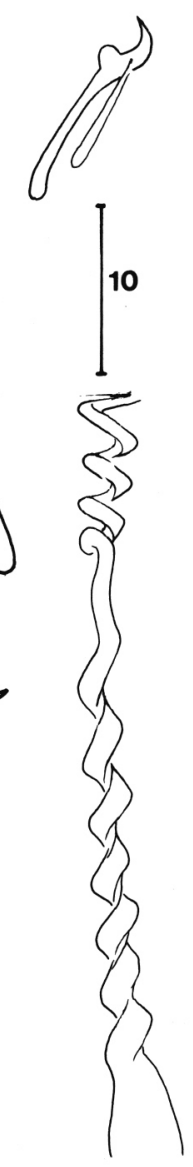

Selon Gèze, 1943, les volcans de la dorsale camerounaise datent de la fin du Quaternaire, leur forme conique et leurs cratères peu altérés militent en faveur de leur jeunesse. D'ailleurs, le Mont Cameroun est toujours actif. La faune ichtyologique dulcicole de l'île Fernando Poo, partie intégrante de la chaîne volcanique camerounaise, atteste de sa formation récente (Thys Van Den Audenaerde, 1967). $\mathrm{Au}$ moment de l'isolement du lac Barombi Mbo, 2 à 4 Cichlidae se seraient trouvés isolés dans un environnement différent de leur biotope originel, avec suffisamment de nourriture et d'espace pour permettre une croissance rapide des populations (Trewavas et al., 1972).

Des observations faites dans des lacs artificiels montrent que dans de telles conditions, les poissons peuvent changer leurs comportements de façon relativement rapide. Le cas de certains Schilbeidae du lac Volta est bien connu (Petr, 1967). Ceci peut expliquer le degré élevé de spéciation des Cichlidae du lac Barombi Mbo. Cependant l'étude des Monogènes de ces poissons ne montre pas un parallélisme entre la spéciation des hôtes et celle de leurs parasites. La 
découverte de $E$. barombiensis n. sp. avec un pénis très spiralé, pose le problème de son origine et, partant, celle de tous les Enterogyrus.

\section{RÉFÉRENCES}

Bilong Bilong C. F., Birgi E., Lambert A. : Enterogyrus melenensis n. sp. (Monogenea, Ancyrocephalidae), parasite stomacal de Hemichromis fasciatus Peters, 1857 (Télostéen, Cichlidae) du Sud-Cameroun. Rev. Zool. Afr. J. Afr. Zool., 1989, 103, 99-105.

Bilong Bilong C. F., Birgi E., Le Brun N., Euzet L. : Urogyrus cichlidarum n. g., N. sp. (Monogenea, Dactylogyridea) parasite de la vessie urinaire de Cichlidae au Cameroun (à paraître).

Euzet L., Prost M. : Report of the meeting on « Monogenea: problems of systemaytics, biology and ecology ». Rev. Adv. Parasitol., 1981, 1003-1004.

Geze B. : Géographie physique et géologique du Cameroun occidental. Mem. Mus. Paris, 1943, 17, 1-272.

Guegan J.-F. : Structure des peuplements parasitaires : le modèle Monogènes de Cyprinidae Ouest-africains. Thèse de Doctorat, Académie de Montpellier, Université Montpellier II, 1990, 1-211.

Gussev A. V. : In: Key to parasites of freshwater fish of the USSR
(Bukhovskaya-Pavlovskaya, I. E.). Akad. Nauk. SSSR, 1962, 919 p. Traduit du russe par IPST, Jérusalem, 1964.

Llewellyn J. : Larvae and larval development of Monogenea. Rev. Adv. Parasitol., 1963, 1, 287-326.

Malmberg G. : The excretory systems and the marginal hooks as a basis for systematics of Gyrodactylus (Trematoda, Monogenea). Arkiv. Zool., 1970, 23, 1-236.

Paperna I. : Studies on the Monogenetic trematodes in Israel. Monogenetic trematodes of Cichlids. Bamidgeh, 1960, 12, 20-30.

Paperna I. : Enterogyrus cichlidarum n. gen. n. sp. A Monogenetic Trematode parasitic in the intestine of a fish. Bull. Res. Counc. Israel, 1963, 11B, 183-187.

Paperna I. : Monogenea of inland water fish in Africa. Ann. Mus. Roy. Afr. Centr., sér. 8 (Zool.), 1979, $\mathrm{n}^{\circ}$ 226, 1-131.

Pariselle A., Lambert A., Euzet L. : A new type of haptor in mesoparasitic monogeneans of the genus Enterogyrus Paperna, 1963: a description of Enterogyrus foratus n. sp. and E. coronatus n. sp., stomach parasites of Cichlidae in West Africa. Systematic Parasitol. (sous presse).

Petr T. : Food-preferences of the commercial fishes of the Volta Lake. Rep. Tech. Ghana Volta basin Res. Proj., 1967, n 10, $1-22$.

Thys Van Den Audernaerde D. F. E. : The freshwater fishes of Fernando Poo. Verk. K. Vlaam. Akad. Wet., 1967, 29, 1-167.

Trewavas E., Green J., Corbet S. A. : Ecological studies on crater lakes in West Cameroon. Fishes of Barombi Mbo. J. Zool. (London), 1972, 167, 41-95. 